\title{
Osteogenesis Imperfecta: The Molecular Basis of Clinical Heterogeneity $^{a}$
}

\author{
PETER H. BYERS, ${ }^{b, c, d, e}$ JEFFREY F. BONADIO, ${ }^{f}$ \\ DANIEL H. COHN, ${ }^{c}$ BARBRA J. STARMAN, ${ }^{c}$ \\ RICHARD J. WENSTRUP, ${ }^{8}$ AND MARCIA C. \\ WILLING ${ }^{d, e}$
}

\author{
Departments of ${ }^{c}$ Pathology and ${ }^{d}$ Medicine \\ and \\ 'Center for Inherited Disease \\ University of Washington \\ Seattle, Washington 98195 \\ ${ }^{f}$ Howard Hughes Medical Institute \\ and \\ Department of Pathology \\ University of Michigan \\ Ann Arbor, Michigan \\ ${ }^{g}$ Department of Pediatrics \\ Duke University \\ Durham, North Carolina 27710
}

During the last six years we have attempted to determine the molecular basis of osteogenesis imperfecta (OI) by analyzing the collagenous proteins synthesized by dermal fibroblasts cultured from affected individuals, their parents, and other family members. During that time we have studied members of more than 400 unrelated families, or, we estimate, between one and five percent of all families with $O I$ in the United States. These studies have, along with those from other laboratories (see REFs. 1,2 for reviews), provided molecular confirmation of the broad outline of clinical classification currently in use, ${ }^{3}$ demonstrated the spectrum of mutations in collagen genes that give rise to clinically distinct forms of $\mathrm{OI}$, helped to understand how mutations result in alterations in collagen metabolism, demonstrated that the study of proteins is an essential first step in the analysis of collagen mutations, and revealed a number of surprises about the nature of inheritance of different forms of OI. In this

${ }^{a}$ Supported in part by grants from the National Institutes of Health (AR 21557, GM 15253, AR 07713, AR 38473, AR 37484) and by a Clinical Research Grant (6-298) from the March of Dimes Birth Defects Foundation.

${ }^{b}$ Address for correspondence: Peter H. Byers, MD, Department of Pathology SM-30, University of Washington, Seattle, WA 98195. 
paper we have detailed our own studies of the molecular basis of clinical heterogeneity in OI, related them to those of others, and tried to present an integrative approach to the relationship between mutation and phenotype.

\section{An Efficient Method to Screen for Mutations in Type I Collagen That Produce Osteogenesis Imperfecta}

The vast majority of cell strains from individuals with osteogenesis imperfecta synthesize either decreased amounts of type I procollagen or molecules that contain chains of altered electrophoretic mobilities. Ordinarily we plate cells at high density (250,000 cells per $35 \mathrm{~mm}$ culture dish), allow them to attach and spread overnight, preincubate for four hours with ascorbate added to medium and then label for about 16 hours with [ ${ }^{3} \mathrm{H}$ ]proline ( 20 to $100 \mu \mathrm{C}$ per dish using $0.7 \mathrm{ml}$ of medium lacking serum). We harvest the medium and cell-layer proteins separately in the presence of protease inhibitors and analyze the pro- $\alpha$ chains under reducing conditions. In addition, we analyze a separate aliquot, following limited proteolysis with pepsin to remove the amino-terminal propeptide extensions, under nonreducing conditions. Together, these allow us to measure the relative amounts of types I and III collagens synthesized (as well as of other collagens), the electrophoretic mobility of the constituent chains, the efficiency of secretion of the molecules, and the efficiency of conversion of procollagen to collagen and to the intermediates in conversion, and to identify the presence of interchain disulfide bonds joining two $\alpha 1(\mathrm{I})$ chains (direct analysis for the presence of cysteine substitutions in the triple-helical domain). This screening system has proved remarkably sensitive to mutations that change the size of the chains and to those which affect mobility because of changes in posttranslational modification. It does not identify mutations that do not affect the rate of synthesis or the electrophoretic mobility of chains.

Perinatal Lethal Osteogenesis Imperfecta (OI Type II)

The First Solution: A Deletion in the COLIA1 Gene

We began our investigations into the molecular basis of $O I$ with cells from an infant, previously studied by Pentinnen et al.," that had a lethal form of OI. They had found that these cells secreted far less type I procollagen than normal cells but did secrete near normal amounts of type III procollagen. ${ }^{4}$ In analyzing the reason for the defective secretion we found that the cells synthesized two species of the pro$\alpha 1$ (I) chain, one that was normal and another that was shorter than normal. The shortened chain was incorporated into molecules efficiently but was secreted from the cells at a very slow rate. Subsequent studies by Williams and Prockop confirmed these findings and located the deletion within the triple-helical domain. ${ }^{6}$ Analysis of mRNA by $S_{1}$ nuclease digestion and of genomic DNA by restriction endonuclease digestion indicated that the protein structure was determined by a multiexon deletion.' The rearrangement in the genomic structure was found, unexpectedly, to be an intron-to- 
intron multiexon deletion that preserved the Gly-X-Y triplet structure while deleting triple-helical residues 327-401 from half the pro- $\alpha 1$ (I) chains. ${ }^{8,9}$ Although these initial successes were provocative and genetically interesting, it was almost immediately clear that rearrangements did not produce the OI type II phenotype in most instances.

\section{Deletions Are Rare, Amino Acid Substitutions Are Common}

Analysis of the type I procollagen molecules synthesized by cell strains from more than 100 infants with OI type II has indicated that the phenotype generally results from apparently subtle mutations that alter the electrophoretic mobility of the molecules without altering their native size..$^{10-12}$ These mutations usually occur in only one of the alleles encoding the chains of type I collagen and, when they have been analyzed, are absent from parental collagens. ${ }^{13}$ The vast majority of cell strains from infants with OI type II synthesize some normal type I procollagen and some molecules the constituent chains of which migrate more slowly than normal. The delay in mobility results from increased posttranslational modification (lysyl hydroxylation and hydroxylysyl glycosylation) of all chains in the affected molecules along all or a part of the triple-helical domain. The increased modification is accompanied by decrease in thermal stability of the triple helix and by decreased efficiency of secretion of the abnormal molecules. ${ }^{10}$

The nature of the subtle mutations that give rise to the dramatic OI type II phenotype was first illustrated by a cell strain identified by Steinmann et al. ${ }^{13}$ in which cysteine was found in the pepsin resistant domain of al(I)CB6 (residues 817-1014 of the triple helix and several residues of the carboxyl-terminal telopeptide). The cell strain synthesized molecules that contained one or two mutant $\alpha 1(\mathrm{I})$ chains and both populations were overmodified. Cohn et al..$^{14}$ isolated portions of both COL1A 1 alleles and determined that the cysteine resulted from a single nucleotide substitution in the codon for glycine at position $\mathbf{9 8 8}$ of the triple helix. On the basis of these findings they suggested that most OI type II cell strains harbored mutations in COL1A1 that resulted in disruption of the Gly-X-Y triple-helical sequence, either by substitutions for glycine (most likely) or by small deletions or insertions that did not change the reading frame but either inserted or deleted amino acids. This prediction has now been confirmed by Bateman et al. ${ }^{15}$ who identified substitution of arginine for glycine at triple-helix position 391 , by Vogel et al. ${ }^{16}$ (glycine 748 to cysteine), and by Constantinou et al. ${ }^{17}$ (glycine 904 to cysteine), with all mutations located in the COL1A1 gene (that encodes the al(I) chain). In those cell strains modification of the chains in the molecules that incorporate the mutant chains is increased amino-terminal to the site of the mutation.

\section{Substitutions Affect Molecular Folding}

We have suggested that the extent of overmodification can be used to determine the approximate location in the chain of the mutant sequence ${ }^{10}$ and that overmodification occurs either because the molecules fail to propagate triple helix beyond the site of the mutation or because the structure of the triple-helix amino-terminal to the 
substitution is altered. The normal winding of triple-helix begins near the carboxylterminal end of the molecule and occurs in a processive fashion until it reaches the amino-terminal end. ${ }^{18}$ Formation of triple helix requires a glycine in every third position and isomerization of cis-peptide bonds at prolyl residues to transpeptide bonds. ${ }^{18}$ The structure is stabilized at $37^{\circ} \mathrm{C}$ by interchain hydrogen bonds involving the amide groups of glycyl residues and the carbonyl groups on adjacent chains; additional stabilization is provided by hydrogen bonding that involves the hydroxyl groups of hydroxyproline. The enzymes that produce posttranslational modification within the triple-helical domain of pro- $\alpha$ chains use only chains in random coil as substrates and the formation of the stable triple helix ordinarily removes the completed molecules from the substrate pool.

There seem to be two classes of possible structural solutions to the question of overmodification. First, triple helix does not propagate beyond the substitution and the chains remain substrates for a substantial time (1-2 hours). Second, although triple helix does propagate beyond the substitution the structure of the molecule formed does not inhibit enzymatic modification (i.e., the molecules have very "loose" triplehelical structures, possibly because the substitution changes "chain order" or in some other way perturbs the normal chain-chain interactions). Whatever the explanation, the excessively modified molecules are not efficiently secreted and some are degraded within the cell. Once these cells reach the extracellular space they probably interfere with the normal processes of fibril formation and of mineralization, although the mechanisms by which these occur is still not understood.

\section{The Nature and Position of the Substitution Determine the Phenotype}

Eight amino acid substitutions can result from single nucleotide changes in a glycine codon (arginine, serine, tryptophan, cysteine, aspartic acid, glutamic acid, valine and alanine). Of these, cysteine has been found in several cell strains (it is easy to identify because of the formation of dimers and thus the nature and location of the mutation is readily detected), arginine has been found in one, and an acidic charge change (presumably due to substitution of aspartic or glutamic acid) has been identified in another. Screening by isoelectric focusing of $\mathrm{CNBr}$ peptides of the chains of type I collagen has identified no additional charge substitutions in about $\mathbf{4 0}$ cell strains. Despite the lack of identification of the precise residue substituted, the extent of overmodification suggests that mutations within a significant portion of the triplehelical domain (most of which are probably in the COL1Al gene) can result in the OI type II phenotype. Substitutions for glycine residues in the triple-helical domain of the $\alpha 1$ (I) chain that result in the OI type II phenotype map between residues 391 (arginine) $)^{15}$ and 988 (cysteine). ${ }^{13,14}$ Radiological-biochemical correlations suggest that when overmodification is most extensive the radiographic picture is worst; ${ }^{10}$ thus, mutations near the carboxyl-terminus of the triple helix are phenotypically most severe.

We think that all substitutions for glycine are not equivalent so that both the nature of the substitution and its position determine the phenotype. The evidence to support such an hypothesis is as follows. First, there is evidence that mutations aminoterminal to residue 391 can produce OI type II $^{10}$ but that other mutations in the same domain, probably substitutions for glycine, can result in the milder OI type III phenotype (see below). Second, substitutions of cysteine for glycine along the $\alpha 1$ (I) chain have markedly different phenotypes varying from the lethal OI type II if the 
substitutions occur at positions 988,901 , or 748 to OI type III phenotype if the substitution occurs at position 526. Finally, there are suggestive data that substitutions further toward the amino-terminal end of the molecule have even less deleterious effects. Thus we think that both the position and the nature of the substituting residue determine the phenotype, in ways that are not yet clear: mutations near the carboxylterminal end are the most severe if they occur in $\alpha 1(I)$ and the "slope" of the phenotypic gradient toward the amino-terminal end may vary with the substituting residue. An alternative explanation for the phenotypic variation is that there are functional, chain-specific domains which are disrupted by the substitutions.

\section{More Rearrangements Have Been Found}

Although rearrangements in the genes of type I collagen are rarely found to result in the OI type II phenotype, we have identified one multiexon deletion from the COL1A2 gene and a multiexon insertion in the COL1A1 gene that each produce the phenotype. These findings suggest that, all told, rearrangements may account for fewer than $5 \%$ of all instances of OI type II.

Cells from the first of these infants synthesized two varieties of the pro- $\alpha 2(1)$ chain, one that was normal and a second that was 180 amino acids shorter than normal by virtue of an intron-to-intron deletion that removed the exons encoding triple-helical residues $586-765 .^{19,20}$ The amino acid deletion predicted by genomic sequence determination leaves the Gly-X-Y triplet structure intact, albeit 180 residues shorter than normal. Molecules that contained the shortened chain were retained within the cell in rough endoplasmic reticulum. Although the assembly of the triple helix in these molecules appeared to start normally, the modification of the triplehelix amino-terminal to the junction was markedly increased. These findings support the concept (see above) that distortion of the normal triple helix may occur if the normal chain-chain interactions are disrupted, even by altering the relative positioning of the charge groups ${ }^{19-22}$ without disrupting the canonical triple-helix Gly-X-Y triplet structure.

The remaining rearrangement we identified was a tandem duplication of an approximately 600 base pair segment of a COL1A 1 allele that resulted in insertion of 50-70 residues in the triple-helical domain of about half the pro-al(I) chains synthesized. ${ }^{23}$ The insertion affected the stability of molecules that incorporated the abnormal chains but we were unable to determine whether the molecules containing one or two of the abnormal chains were least stable.

\section{Clinical Genetics of the OI Type II Phenotype: Germinal Mosaicism Offers an Alternative Explanation for Recurrence in Families}

When initially segregated as a distinct variety of OI, the perinatal lethal phenotype was thought to represent an autosomal recessive disorder, ${ }^{3}$ an idea that was supported by presentation of additional families in which there had been recurrence or in which the proband was born to consanguineous parents. ${ }^{24}$ The biochemical data presented by our group and by others and the more recent clinical genetic studies ${ }^{10.25}$ were, 
however, most consistent with infants with the OI type II phenotype resulting from new dominant mutations. For example, Thompson and her colleagues ${ }^{23}$ identified no recurrences in 30 families. In our own study we determined an empiric recurrence risk of $6.7 \% .^{12}$ Biochemical studies of the collagens synthesized by cells grown from several of the infants in the recurrent families indicated that they were indistinguishable from those of the sporadic varieties in which the mutation that was the basis of the phenotype had been determined. These studies were most compatible with one parent in each of these families being mosaic in the germ line for a population of cells that carried the mutation. The structure of some of the families (that is, men or women who had more than one affected infant with different partners) is compatible with this concept. We think that germinal mosaicism is the best explanation for recurrence of the OI type II phenotype although we cannot exclude the possibility of autosomal recessive disease in rare families.

\section{Mild to Moderate Dominantly Inherited Forms of Osteogenesis Imperfecta (OI Type I and OI Type IV)}

\section{Biochemical Heterogeneity}

In the course of examining collagens synthesized by cells from patients with mild dominantly inherited forms of OI two patterns emerged: those from patients with $\mathrm{OI}$ type I synthesized about half the amount of normal type I procollagen while those from OI type IV patients synthesized some normal type I procollagen and some molecules that, like those synthesized by cells from infants with OI type II, were overmodified along a portion of the triple helix.

OI Type I Results from Mutations That Affect the "Expression" of Type I Collagen Genes

Cells cultured from most individuals with OI type I synthesize normal amounts of type III procollagen but secrete about half the normal amount of type I procollagen. ${ }^{26}$ In three cell strains previously studied the decrease in secretion of type I procollagen resulted from decreased synthesis of pro- $\alpha 1(I)$ chains. Because the chains of type I procollagen can be assembled as trimers that contain either two pro- $\alpha$ l(I) chains and a single pro- $\alpha 2(\mathrm{I})$ chain or three pro- $\alpha 1(\mathrm{I})$ chains, synthesis of half the normal amount of pro- $\alpha 1$ (I) allows secretion of only half normal amounts of type I procollagen. The remaining pro- $\alpha 2(\mathrm{I})$ is degraded. Analysis of six cell strains from individuals with OI type I indicates that both COL1A1 alleles are intact suggesting that deletion of the entire gene is an uncommon event. ${ }^{27}$ Because mRNA levels are also decreased in some of these cell strains it is likely that promoter and splicing mutations will commonly be found to result in OI type $\mathrm{I},{ }^{28,29}$ as might be expected with the highly complex structure of the genes that encode the chains of type I collagen. ${ }^{30}$

The biochemical data are consistent with the "functional deletion" of a COL1A1 allele and thus in large part support the linkage studies which suggest that the OI 
type I phenotype usually results from mutations in the COL1AI gene. None of the linkage studies ${ }^{31}$ has identified loss of those portions of the gene used to determine linkage, again supporting the concept that more subtle mutations can result in marked loss of function of an allele.

\section{OI Type IV Is Heterogenous}

Most cell strains that we have examined from individuals with OI type IV produce two populations of type I procollagen molecules, those that are normal and those that are overmodified along a portion of the triple-helical domain. In contrast to cells from infants with OI type II, the abnormal procollagens are generally secreted quite efficiently even though the extent of modification may be similar. ${ }^{32}$

Wenstrup and colleagues ${ }^{32}$ identified the first of these infants, a child with mild short stature, bowed femurs and rare fractures, born to normal parents. The cells from the parents synthesized only normal type I procollagen molecules while those from the child synthesized normal molecules and those that were overmodified along the full length of the chain. The thermal stability of the overmodified molecules was decreased similar to molecules synthesized by cells from infants with OI type II. These findings indicated that the phenotype in the child resulted from a new mutation in one of the genes of type I collagen but the identity of the chain in which the mutation occurred could not be determined. Given the difference in behavior of the molecules and the difference in clinical phenotype, we thought it probably resulted from a mutation in a COL1A2 allele.

To further our understanding of this phenotype we undertook several additional investigations: first, the analysis of defects in families with OI type IV in which linkage assigned the defect to the COL1A2 gene; second, analysis of individuals whose cells synthesized overmodified type I collagen molecules; and, finally, a deliberate search for point mutations that result in the appearance of cysteine within the triple-helical domain of $\alpha 2(\mathrm{I})$.

\section{OI Type IV Linked to COL1A2, a Surprising Biochemical Finding}

We began our search with cells from patients in a dominant family in which linkage to COL1A2 had been demonstrated with high likelihood. ${ }^{33}$ Cells from the unaffected family members synthesized only normal collagens while those from the affected individuals synthesized and secreted normal type I and retained an overmodified species of type I procollagen that they appeared not to secrete at all. Two-dimensional peptide mapping of the retained type I procollagen indicated that some of the $\alpha 2(\mathrm{I})$ chains were shorter than normal by virtue of deletion of 10-20 residues located between residues 358 and $776 .^{34}$ The deletion resulted in overmodification of the pro- $\alpha 1$ (I) chains contained in the same molecules amino-terminal to the domain of the deletion. This cell strain presented uncertainties, the most pressing of which was how secretion of only normal molecules could result in this phenotype. Such questions remain unresolved and the nature of the mutation has not yet been determined. 


\section{Amino Acid Substitutions in a2(I) Result in the OI Type IV Phenotype}

One of the cell strains most helpful to the understanding of the molecular basis of the OI type IV phenotype originated from an individual in a small family in which the phenotype was inherited in an autosomal dominant fashion. ${ }^{35}$ Cells from the affected individuals synthesized and secreted normal type I procollagen molecules and others which were overmodified along the entire length of the chains. Despite the increased modification the thermal stability of the abnormal molecules was normal (thus distinguishing the effect of overmodification from stability). Separation of $\mathrm{CNBr}$ fragments of fibroblast collagenase-digested type I collagen, first by isoelectric focussing and then by size, demonstrated a basic charge change in the B-fragment of some of the $\alpha 2(\mathrm{I})$ chains. Portions of both COL1A2 alleles were isolated using a cosmid vector $^{20}$ and the nucleotide sequence of the exons encoding the domain carrying the charge change was determined. The mutant allele (determined by linkage analysis in the family) contained a single nucleotide change that resulted in substitution of arginine for the last glycine of the triple-helical domain (position 1012). The glycine at position 1012 is the first "triple-helical" glycine encountered in the propagation of structure from the carboxyl-terminal end of the molecule. Interestingly, the effect on modification is striking although the thermal stability of the molecules is normal. This argues that extensive overmodification or the structural defect itself, first, must alter the assimilation of molecules into a matrix and, second, may interfere with normal matrix mineralization. The molecular findings in this family confirmed the hypothesis that mutations in the COL1A1 and COL1A2 genes result in different phenotypes, presumably a reflection of the functional differences between the chains. Finally, these findings suggested that point mutations that result in substitutions for glycine in the $\alpha 2$ (I) chain are likely to be common features of the OI type IV phenotype.

\section{Cysteine in the Triple-Helical Domain of a2(I) Confirms That Point Mutations in COL1A2 Are Common Features of the OI Type IV Phenotype}

Because of the size of the $\mathrm{CNBr}$ peptides of the $\alpha 2(\mathrm{I})$ chain and the difficulty in cleaving the methionyl residue that separates the large peptides $\alpha 2$ (I)CB3 and $\alpha 2(I) C B 5$ it is difficult to identify charge mutations in the chain. To assess how frequently point mutations (rather than small deletions or insertions) are likely to account for the OI type IV phenotype, we analyzed cell strains from 60 different patients with the condition for the presence of cysteine in the triple helix. ${ }^{36}$ Cells were labeled with $\left[{ }^{35} \mathrm{~S}\right]$ cysteine and the proteins secreted into the medium were digested with pepsin to remove the cysteine-containing propeptide extensions, and the $\alpha$ chainsize fragments were analyzed by gel electrophoresis. We found four cell strains in which a2(I) labeled with cysteine. In one the residue was located in a2(I)CB4, it was absent from type I collagen chains synthesized by both parental cell strains, the amino-terminal portion of type I collagen molecules was overmodified, and the phenotype was similar to OI type III. In the other three instances, the cysteine was located between residues 357 and 776, the type I collagen molecules that contained the mutant chain were overmodified along about half the length of the triple helix and, with one exception, the phenotype was mild to moderate in severity. These findings confirmed that the OI type IV phenotype commonly results from point mutations that, most likely, result in substitutions for glycine in the triple-helical domain of a2(I). 


\section{Substitutions in the al(I) Chain Can Produce the OI Type IV Phenotype}

Linkage data from Sykes et $a l^{31}$ and Wallis et $a .^{37}$ suggest that the OI type IV phenotype is genetically heterogeneous. We have identified one family with OI type IV cells from affected members of which synthesize normal pro- $\alpha 1(\mathrm{I})$ chains and others with a cysteine near residue 400 of the triple-helical domain, ${ }^{38}$ probably similar to the family described by deVries and de Wet. ${ }^{39}$

\section{Progressive Deforming Osteogenesis Imperfecta (OI Type III)}

\section{OI Type III Often Results from Dominant Mutations}

Despite the clinical severity, OI type III has been a difficult phenotype to define biochemically. The difficulty has its origin in both genetic heterogeneity and the nature of mutations in the dominantly inherited forms of the phenotype. When originally described OI type III was thought to be inherited in an autosomal recessive fashion, ${ }^{3}$ a concept supported, in part, by the more frequent consanguinity of parents. ${ }^{40}$ Although the evidence to support recessive inheritance in some individuals with such a phenotype is excellent, ${ }^{41-43}$ from a genetic point of view it is likely that many individuals with the phenotype represent new dominant mutations. The recurrence risk in families with an affected child is small and a few affected individuals have had affected children, consistent with the condition resulting from dominantly inherited mutations.

\section{Point Mutations Can Produce the OI Type III Phenotype}

We have now identified three individuals with apparent point mutations that result in amino acid substitutions in the $\alpha 1$ (I) or $\alpha 2(I)$ chains of type I collagen and produce the OI type III phenotype. The first is a substitution of cysteine for the glycine at position 526 of the triple-helical domain of $\alpha 1(\mathrm{I}){ }^{44}$ As indicated above, this substitution in the $\alpha$ l(I) chain lies between glycine residues 391 and 988 for which substitutions produce the more severe OI type II phenotype. We think that cysteine residues may be less deleterious substitutions, provided they are toward the amino-terminal half of the chain, because of the potential of stabilizing intramolecular, interchain disulfide bonds. Alternatively, it is possible that glycine 526 is in a less essential location of the $a 1$ (I) chain than the other residues at which substitution is lethal. In one other patient we have identified a charge change in al(I)CB8 (residues 124-401 of the triple helix) which does not result in significant overmodification of the peptide. The lack of significant overmodification suggests that the substitution is near the aminoterminal end of the peptide. Finally, we have identified a cysteine within $\alpha 2$ (I)CB4 that also results in the OI type III phenotype and produces overmodification of a portion of the molecules that incorporate the mutant chain.

These last two mutations are all near the amino-terminal end of the molecule and do not have major effects on posttranslational modification or on secretion. As a result, they can be detected only by detailed analysis of protein structure and are not readily 
detected by most methods of screening cell strains at the protein level. Such mutations, as well as the possibility that some forms of OI type III could result from noncollagen mutations, probably explains the difficulty in identifying the molecular basis of this form of OI.

\section{A Unifying Theme}

At first glance the myriad of mutations that give rise to the different phenotypes of osteogenesis imperfecta defies a unitary explanation. However, as the patterns engendered by the bulk of the mutations are examined more closely a number of themes emerge. First, mutations that affect the expression of the COL1Al gene (and probably the COL1A2 gene) are milder in their phenotypic effects than those which affect the structure of the protein produced thus suggesting that the structurally abnormal molecules contribute to the bone matrix. Second, point mutations in the COL1Al gene that result in substitutions for glycine residues in the triple helix are, in general, much more severe than those which affect the COL1A2 gene. We think this may result from two considerations: the role of $\alpha 1(\mathrm{I})$ in a molecule may be different than that of $a 2(I)$, and more molecules will be abnormal if mutations affect an $\alpha 1$ (I) chain than if they affect an $\alpha 2(\mathrm{I})$ chain. Third, there is a gradient of effect on molecules as mutations are moved from the carboxyl-terminal end toward the amino-terminal end; this gradient is reflected in clinical severity and is probably modified by the nature of the amino acid that substitutes for glycine. Fourth, large rearrangements in either of the genes of type I collagen produce the lethal phenotype although small deletions may vary in their phenotypic expression depending on the chain and the location in the chain of the mutation. Finally, most forms of OI result from dominant mutations.

On the basis of our observations and those from other laboratories it has become possible to predict the nature of the mutation given the clinical phenotype. Despite the evolution of our understanding of the nature of the mutations, we still have little understanding of how the mutations are translated into a phenotype or why, given the same mutation, the phenotype may differ radically among family members. Nonetheless, these studies have served, in large part, to confirm and expand the basic clinical classification of OI, to provide a more rational basis for genetic counseling, and to begin to understand the molecular basis of this complex and heterogeneous disorder.

\section{REFERENCES}

1. ByeRS, P. H. \& J. F. BonAdio. 1985. The molecular basis of clinical heterogeneity in osteogenesis imperfecta: mutations in type I collagen genes have different effects on collagen processing. In Genetic and Metabolic Disease in Pediatrics. J. K. Lloyd \& C. R. Scriver, Eds. 56-90. Butterworth. London.

2. Prockop, D. J. \& K. I. KivirikKo. 1984. Heritable diseases of collagen. N. Engl. J. Med. 311: 376-386.

3. Sillence, D. O., A. S. SENN \& D. M. DANKS. 1979. Genetic heterogeneity in osteogenesis imperfecta. J. Med. Genet. 16: 101-106.

4. Pentinnen, R. P., J. R. Lichtenstein, G. R. Martin \& V. A. McKusick. 1975. 
Abnormal collagen metabolism in cultured cells in osteogenesis imperfecta. Proc. Natl. Acad. Sci. USA 72: 586-589.

5. BARSH, G. S. \& P. H. BYERS. 1981. Reduced secretion of structurally abnormal type I procollagen in a form of osteogenesis imperfecta. Proc. Natl. Acad. Sci. USA 78: 5142-5146.

6. Williams, C. J. \& D. J. Prockop. 1983. Synthesis and processing of a type I procollagen containing shortened proal(I) chains by fibroblasts from a patient with osteogenesis imperfecta. J. Biol. Chem. 258: 5915-5921.

7. Chu, M.-L., C. J. Williams, G. Pepe, J. L. Hirsch, D. J. Prockop \& F. Ramirez. 1984. Internal deletion in a collagen gene in a perinatal lethal form of osteogenesis imperfecta. Nature 304: 78-80.

8. Chu, M.-L., V. Gargiulo, C. Williams \& F. Ramirez. 1985. Multiexon deletion in an osteogenesis imperfecta variant with increased type III collagen mRNA. J. Biol. Chem. 260: 691-694.

9. Barsh, G. S., C. L. Roush, J. Bonadio, P. H. Byers \& R. E. Gelinas. 1985. Intronmediated recombination may cause a deletion in an al type I collagen chain in a lethal form of osteogenesis imperfecta. Proc. Natl. Acad. Sci. USA 82: 2870-2874.

10. BonAdio, J. F. \& P. H. BYERS. 1985. Subtle structural alterations in the chains of type I procollagen produce osteogenesis imperfecta type II. Nature 316: 363-366.

11. Bateman, J. F., T. Mascara, D. Chan \& W. G. Cole. 1984. Abnormal type I collagen metabolism by cultured fibroblasts in lethal perinatal osteogenesis imperfecta. Biochem. J. 217: 103-115.

12. Byers, P. H., P. Tsipouras, J. F. Bonadio, B. J. Starman \& R. C. Schwartz. 1988. Perinatal lethal osteogenesis imperfecta (OI type II): a biochemically heterogeneous disorder usually due to new mutations in the genes for type I collagen. Am. J. Hum. Genet. 42: 237-248.

13. Steinmann, B., V. H. Rao, A. Vogel, P. Bruckner, R. Gitzelmann \& P. H. Byers. 1984. Cysteine in the triple-helical domain of one allelic product of the al(1) gene of type I collagen produces a lethal form of osteogenesis imperfecta. J. Biol. Chem. 259: 11129-11138.

14. Cohn, D. H., P. H. Byers, B. Steinmann \& R. E. Gelinas. 1986. Lethal osteogenesis imperfecta resulting from a single nucleotide change in one human pro- $\alpha 1(\mathrm{I})$ collagen allele. Proc. Natl. Acad. Sci. USA 83: 6045-6047.

15. Bateman, J. F., D. Chan, I. D. Walker, J. G. Rogers \& W. G. Cole. 1987. Lethal perinatal osteogenesis imperfecta due to substitution of arginine for glycine at residue 391 of the a I(I) chain of type I collagen. J. Biol. Chem. 262: 7021-7027.

16. Vogel, B. E., R. R. MinoR, M. Freund \& D. J. Prockop. 1987. A point mutation in a type I procollagen gene converts glycine 748 of the $\alpha 1$ chain to cysteine and destabilizes the triple helix in a lethal variant of osteogenesis imperfecta. J. Biol. Chem. 262: $14737-14744$.

17. Constantinou, C. D., K. B. Nielsen \& D. J. Prockop. 1988. The molecular defect in a lethal variant of osteogenesis imperfecta is a single base mutation that substitutes cysteine for glycine 904 of the $\alpha$ (I) chain of type I procollagen. Submitted.

18. Bachinger, H. P., P. Bruckner, R. Timpl, D. J. Prockop \& J. Engel. 1980. Folding mechanism of the triple helix in type-III collagen and type-III pN-collagen. Eur. J. Biochem. 106: 619-632.

19. Willing, M. C., D. H. Cohn, B. J. Starman, K. A. Holbrook, C. R. Greenberg \& P. H. BYERs. 1988. Heterozygosity for a large deletion in the a2(I) collagen gene (COL1A2) has a dramatic effect on type I collagen secretion and produces perinatal lethal osteogenesis imperfecta. J. Biol. Chem. 263: 8398-8404.

20. Cohn, D. H., R. J. Wenstrup, M. C. Willing, J. F. Bonadio \& P. H. Byers. 1988. General strategies for isolating the genes encoding type I collagen and for characterizing mutations which produce osteogenesis imperfecta. Ann. N. Y. Acad. Sci. This volume.

21. RAUTERBERG, J. \& K. KUHN. 1968. The renaturation behaviour of modified collagen molecules. Hoppe-Seyler's Z. Physiol. Chem. 349: 611-622.

22. Salem, G. \& W. Traub. 1975. Conformational implications of amino acid sequence regularities in collagen. FEBS Lett. 51: 94-99. 
23. Byers, P. H., B. J. Starman, D. H. CoHn \& P. H. Byers. 1988. A novel mutation causes a perinatal lethal form of osteogenesis imperfecta: an insertion in one a1(I) collagen allele (COL1A1). J. Biol. Chem. 263: 7855-7861.

24. Sillence, D. O., K. K. Barlow, A. P. Barber, J. G. Hall \& D. L. Rimoin. Osteogenesis imperfecta type II. Delineation of the phenotype with reference to genetic heterogeneity. Am. J. Med. Genet. 17: 407-423.

25. Thompson, E. M., I. D. Young, C. M. Hall \& M. E. Pemberq. 1987. Osteogenesis imperfecta type IIA: evidence for dominant inheritance. J. Med. Genet. 24: 386-389.

26. BARSH, G. S., K. E. DAvid \& P. H. BYERS. 1982. Type I osteogenesis imperfecta: a nonfunctional allele for proal(I) chains of type I procollagen. Proc. Natl. Acad. Sci. USA 79: 3838-3842.

27. WILling, M. C., D. H. CoHN \& P. H. BYERs, unpublished observations.

28. Rowe, D. W., J. R. Shapiro, M. Poirier \& S. Schlesinger. 1985. Diminished type I collagen synthesis and reduced alpha $1(\mathrm{I})$ collagen messenger RNA in cultured fibroblasts from patients with dominantly inherited (type I) osteogenesis imperfecta. J. Clin. Invest. 71: $689-697$.

29. GENOVESE, C. \& D. RowE. 1987. Analysis of cytoplasmic and nuclear messenger RNA in fibroblasts from patients with type I osteogenesis imperfecta. Methods Enzymol. 145: 223-235.

30. De Wet, W., M. Bernard, V. Benson-Chanda, M.-L. Chu, L. Dickson, D. Weil \& F. RAMIREZ. 1987. Organization of the human pro- $\alpha 2(\mathrm{I})$ collagen gene. J. Biol. Chem. 262: $16032-16036$.

31. Sykes, B., D. OgILvie, P. Wordsworth, J. ANDERson \& N. Jones. 1986. Osteogenesis imperfecta is linked to both type I collagen structural genes. Lancet 1: 69-72.

32. Wenstrup, R. J., A. G. W. Hunter \& P. H. Byers. 1986. Osteogenesis imperfecta type IV: evidence of abnormal triple helical structure of type I collagen. Hum. Genet. 74: 47-53.

33. Tsipouras, P., J. C. Myers \& F. Ramirez. 1983. Restriction fragment length polymorphism associated with the proa2(I) gene of human type I procollagen. J. Clin. Invest. 72: $1262-1267$.

34. Wenstrup, R. J., P. Tsipouras \& P. H. Byers. 1986. Osteogenesis imperfecta type IV: biochemical confirmation of genetic linkage to the proa2(I) gene of type I collagen. J. Clin. Invest. 78: 1449-1455.

35. Wenstrup, R. J., D. H. Cohn, T. COHEN \& P. H. Byers. 1988. Arginine for glycine substitution in the triple helical domain of the products of one a2(I) collagen allele (COL1A2) produces the osteogenesis imperfecta type IV phenotype. J. Biol. Chem. 263: 7734-7740.

36. COHN, D. H. \& P. H. BYERS. In preparation.

37. Wallis, G., P. Beighton, C. Boyd \& C. Mathew. 1986. Mutations associated with the proa2(I) collagen gene are responsible for several cases of osteogenesis imperfecta. J. Med. Genet. 23: 411-416.

38. Byers, P. H. \& B. J. STARMAN. In preparation.

39. DEVRIES, W. N. \& W. J. DE WET. 1986. The molecular defect in an autosomal dominant form of osteogenesis imperfecta. Synthesis of type I procollagen containing cysteine in the triple-helical domain of pro- $\alpha$ I(I) chains. J. Biol. Chem. 261: 9056-9064.

40. Sillence, D. O., K. K. Barlow, W. G. Cole, S. Dietrich, A. P. Garber \& D. L. RIMOIN. 1986. Osteogenesis imperfecta type III: delineation of the phenotype with reference to genetic heterogeneity. Am. J. Med. Genet. 23: 821-832.

41. Nicholls, A. C., F. M. POPE \& H. SChLOON. 1979. Biochemical heterogeneity of osteogenesis imperfecta: a new variant. Lancet 1: 1193.

42. DeAk, S. B., A. Nicholls, F. M. Pope \& D. J. Prockop. 1983. The molecular defect in a nonlethal variant of osteogenesis imperfecta. Synthesis of proa2(I) chains which are not incorporated into trimers of type I procollagen. J. Biol. Chem. 258: 15192-15197.

43. Dickson, L. A., T. Pihlajaniemi, S. Deak, F. M. Pope, A. Nicholls, D. J. Prockop \& J. C. MYERS. 1984. Nuclease $S_{1}$ mapping of a homozygous mutation in the carboxypropeptide coding region of the proa2(I) collagen gene in a patient with osteogenesis imperfecta. Proc. Natl. Acad. Sci. USA 81: 4524-4528.

44. Starman, B. J., D. H. Cohn, L. Weiss, S. Apone, D. Eyre \& P. H. Byers. In preparation. 